\title{
Successful Sirolimus Treatment for Korean Patients with Activated Phosphoinositide 3-kinase $\delta$ Syndrome 1: the First Case Series in Korea
}

\author{
Ji-Man Kang, ${ }^{1,2 *}$, Su Kyung Kim ${ }^{3 *}$, Dongsub Kim ${ }^{3,4}$, Sae Rom $\mathrm{Choi}^{3}$, Yeon Jung $\mathrm{Lim}^{5}$, \\ Soon Ki Kim ${ }^{6}$, Byung-Kiu Park ${ }^{7}$, Weon Seo Park ${ }^{8}$, Eun-Suk Kang ${ }^{9}$, \\ Young Hyeh $\mathrm{Ko}^{10}$, Yon Ho Choe ${ }^{3}$, Ji Won Lee ${ }^{3}$, and Yae-Jean $\mathrm{Kim}^{3}$ \\ ${ }^{1}$ Department of Pediatrics, Severance Children's Hospital, Yonsei University College of Medicine, Seoul; \\ ${ }^{2}$ Institute for Immunology and Immunological Diseases, Yonsei University College of Medicine, Seoul; \\ ${ }^{3}$ Department of Pediatrics, Sungkyunkwan University School of Medicine, Samsung Medical Center, Seoul; \\ ${ }^{4}$ Department of Pediatrics, Kyungpook National University Hospital, School of Medicine, Kyungpook National University, Daegu; \\ ${ }^{5}$ Department of Pediatrics, College of Medicine, Chungnam National University, Daejeon; \\ ${ }^{6}$ Department of Pediatrics, Inha University Medical Center, Incheon; \\ ${ }^{7}$ Center for Pediatric Cancer, National Cancer Center, Goyang; \\ ${ }^{8}$ Department of Pathology, National Cancer Center, Goyang; \\ ${ }^{9}$ Department of Laboratory Medicine and Genetics, Sungkyunkwan University School of Medicine, Samsung Medical Center, Seoul; \\ ${ }^{10}$ Department of Pathology, Sungkyunkwan University School of Medicine, Samsung Medical Center, Seoul, Korea.
}

Activated phosphoinositide 3-kinase $\delta$ syndrome (APDS)1 is caused by gain-of-function mutations in PIK3CD, which encodes the catalytic p1 $10 \delta$ subunit of phosphoinositide 3 kinase. We describe three patients with APDS1, the first thereof in Korea. Therein, we investigated clinical manifestations of APDS1 and collected data on the efficacy and safety profile of sirolimus, a mammalian target of rapamycin inhibitor and pathway-specific targeted medicine. The same heterozygous PIK3CD mutation was detected in all three patients (E1021K). After genetic diagnosis, all patients received sirolimus and experienced an excellent response, including amelioration of lymphoproliferation and improvement of nodular mucosal lymphoid hyperplasia in the gastrointestinal tract. The median trough level of sirolimus was $5.5 \mathrm{ng} / \mathrm{mL}$ (range, 2.8-7.5) at a dose of $2.6-3.6 \mathrm{mg} / \mathrm{m}^{2}$. Two patients who needed highdose, short-interval, immunoglobulin-replacement treatment (IGRT) had a reduced requirement for IGRT after initiating sirolimus, and the dosing interval was extended from 2 and 3 weeks to 4 weeks. The IgG trough level after sirolimus treatment (median, $594 \mathrm{mg} / \mathrm{dL}$; range, 332-799 mg/dL) was significantly higher than that before sirolimus treatment (median, $290 \mathrm{mg} / \mathrm{dL}$; range, $163-346 \mathrm{mg} / \mathrm{dL})(p<0.001)$. One episode of elevated serum creatinine with a surge of sirolimus (Patient 2$)$ and episodes of neutropenia and oral stomatitis (Patient 1) were observed. We diagnosed the first three patients with APDS1 in Korea. Low-dose sirolimus may alleviate clinical manifestations thereof, including hypogammaglobulinemia.

Key Words: Activated phosphoinositide 3-kinase delta syndrome1, sirolimus, Korea

Received: March 20, 2020 Revised: April 13, 2020 Accepted: April 13, 2020

Corresponding author: Yae-Jean Kim, MD, PhD, Department of Pediatrics, Sungkyunkwan University School of Medicine, Samsung Medical Center, 81 Irwon-ro, Gangnam-gu, Seoul 06351, Korea.

Tel: 82-2-3410-3539, Fax: 82-2-3410-0043, E-mail: yaejeankim@skku.edu

*Ji-Man Kang and Su Kyung Kim contributed equally to this work.

- The authors have no potential conflicts of interest to disclose.

(C) Copyright: Yonsei University College of Medicine 2020

This is an Open Access article distributed under the terms of the Creative Commons Attribution Non-Commercial License (https://creativecommons.org/licenses/by-nc/4.0) which permits unrestricted non-commercial use, distribution, and reproduction in any medium, provided the original work is properly cited. 
Activated phosphoinositide 3 -kinase $\delta$ syndrome (APDS) is a primary immunodeficiency (PID) caused by hyperactivity of phosphatidylinositol-3-OH kinase. ${ }^{1,2}$ APDS1 is caused by gainof-function mutations in PIK3CD, and APDS2 is caused by lossof-function mutations in PIK3R1.,4 Both gene mutations result in hyperactivity of the PI3K/Akt/mammalian target of rapamycin (mTOR) signaling pathway, leading to lymphoproliferation and immunosuppression. ${ }^{5,6}$ Here, we describe three patients with a heterozygous $P I K 3 C D$ mutation treated with sirolimus, an mTOR inhibitor, which alleviated the hypogammaglobulinemia, lymphoproliferation, and enteropathy. This study was approved by the Institutional Review Board of Samsung Medical Center (IRB File No.2018-0013).

A 4-year-old male (Patient 1) diagnosed with common variable immunodeficiency (CVID) was referred to our clinic for persistent lymph node enlargement, hematochezia, and increased serum immunoglobulin (Ig)M. His medical history included double barrel enterostomy and segmental resection of the small intestine, attributable to intestinal malrotation and perforation in the neonatal period. He was diagnosed with hemolytic anemia at the age of 10 months and pancytopenia at the age of 15 months, after which he received steroid treatment. At the age of 21 months, he exhibited increased IgM (304 mg/dL; normal, 43-173 mg/dL) and decreased IgG (16 mg/dL; normal, 345-1236 mg/dL) and IgA (<1 mg/dL; normal, 11-106 mg/dL). Regular immunoglobulin-replacement treatment (IGRT, 500 $\mathrm{mg} / \mathrm{kg}$ every 3 weeks) was initiated for CVID. At the age of 10 months, a lymph node biopsy revealed atypical lymphoid cell proliferation. At the age of 3 years, hematochezia gradually increased, and IgG trough levels were not well maintained (average, $333 \mathrm{mg} / \mathrm{dL}$; normal, 345-1236 mg/dL) despite IGRT (500 $\mathrm{mg} / \mathrm{kg}$ every 3 weeks). Patient 1 had a head circumference of $55 \mathrm{~cm}$ (>95th percentile) and body weight of $15 \mathrm{~kg}(<10$ th percentile), as well as multiple enlarged cervical lymph nodes and splenomegaly. Complete blood count values were white blood cells (WBC), 7910/ $\mu \mathrm{L}$ (normal, 6000-15000/ $\mu \mathrm{L}$ ); hemoglobin (Hb), $10.9 \mathrm{~g} / \mathrm{dL}$ (normal, 10.5-14.0 g/dL); and platelets (PLT), $122 \mathrm{k} / \mu \mathrm{L}$ (normal, 150-450 k/ $\mu \mathrm{L}$ ). Immunological values were CD3, 68\%, 2320/ $\mu \mathrm{L}$ (normal: 56-75\%, 1400-3700/ $\mu \mathrm{L}$ ); CD4, 19\%, 648/ $\mu \mathrm{L}$ (normal: 28-47\%, 700-2200/ $\mu \mathrm{L}$ ); CD8, 45\%, 1535/ $\mu \mathrm{L}$ (normal: 16-30\%, 490-1300/ $\mu \mathrm{L}$ ); and CD19, 10\%, 341/ $\mu \mathrm{L}$ (normal: 8-39\%, 180-1300/ $\mu \mathrm{L})$. Serum IgG, A, and M levels were $332 \mathrm{mg} / \mathrm{dL}$ (normal, 345-1236 mg/dL), <1 mg/dL (normal, 14$159 \mathrm{mg} / \mathrm{dL}$ ), and $569 \mathrm{mg} / \mathrm{dL}$ (normal, 43-207 mg/dL), respectively (Table 1). Mucosal nodular lymphoid hyperplasia visualized as cobblestone-like polyps and cytomegalovirus (CMV) was detected in a mucosal biopsy through colonoscopy. Diagnostic exome sequencing results revealed a heterozygous E1021K mutation in PIK3CD, which was confirmed via Sanger sequencing. After treatment with oral valganciclovir (VGCV) for 6 weeks, follow-up colonoscopy results showed eradication of CMV in the colon, but mucosal nodular lymphoid hyperplasia and hematochezia persisted. Subsequently, sirolimus was started, and trough sirolimus levels were maintained around $5 \mathrm{ng} / \mathrm{mL}$ at a dose of $2 \mathrm{mg}\left(3.0 \mathrm{mg} / \mathrm{m}^{2}\right)$. Upon treatment, diarrheal episodes decreased, weight increased, and lymph node enlargement disappeared. After 6 months of treatment, a follow-up colonoscopy showed improved mucosal nodular lymphoid hyperplasia and decreased cobblestone lesions on the duodenal bulb. Currently, he is receiving regular follow-up visits and has no signs of major invasive infection or any complications. However, the low IgG level has not fully recovered. Therefore, IGRT is regularly administered with prolonged intervals (extended from $800 \mathrm{mg} / \mathrm{kg}$ every 2 weeks to $800 \mathrm{mg} / \mathrm{kg}$ every 4 weeks) (Fig. 1G). Neutropenia and oral stomatitis improved during sirolimus treatment after administering intermittent granulocyte colony-stimulating factor. No other serious adverse events were noted.

A 4-year-old male (Patient 2) with a history of idiopathic thrombocytopenic purpura, Bacillus Calmette-Guérin vaccine site infection, and respiratory tract infection requiring hospitalization was referred to our clinic for enlargement of multiple lymph nodes suspicious for lymphoma. Cervical lymph node enlargement was first noticed at 31 months old. At the age of 3 years, decreased IgG (89 mg/dL) with elevated IgM (347 mg/dL) and normal IgA (16 mg/dL) were detected at a prior hospital. He received regular IGRT under the interim diagnosis of CVID; however, trough IgG levels remained below $400 \mathrm{mg} / \mathrm{dL}$ despite receiving a higher IGRT dose $(800 \mathrm{mg} / \mathrm{kg})$ every 3 weeks. Patient 2 had a head circumference of $52.8 \mathrm{~cm}$ (95th percentile) and body weight of $17 \mathrm{~kg}$ (25-50th percentile). Multiple enlarged lymph nodes in the cervical, supraclavicular, and inguinal areas were palpated, and massive splenomegaly was noted. Molloscum contagiosum was observed on his buttocks. Complete blood count results included WBC, 3300/ $\mu \mathrm{L} ; \mathrm{Hb}, 13.1 \mathrm{~g} / \mathrm{dL}$; and PLT, $157 \mathrm{k} / \mu \mathrm{L}$. Immunological values were CD3, $867 / \mu \mathrm{L}$ (normal, 1578-3707/ $\mu \mathrm{L}$ ); CD4, 241/ $\mu \mathrm{L}$ (normal, 870-2144/ $\mu \mathrm{L}$ ); CD8, 613/ $\mu \mathrm{L}$ (normal, 472-1107/ $\mu \mathrm{L}$ ); and CD19, 24/ $\mu \mathrm{L}$ (normal, 276-640/ $\mu \mathrm{L}$ ). The CD4:CD8 ratio was 0.39 (Table 1). Positron emission tomography/computed tomography (PET/CT) findings showed enlargement of multiple lymph nodes, including the bilateral cervical, supraclavicular, and inguinal areas, and abnormal uptake in the whole intestine. A colonoscopy revealed multiple nodules and masses throughout the entire length of the colon. Cervical lymph node and large intestine biopsies revealed atypical lymphocyte proliferation with polyclonality (Fig. 1A-C) (Supplementary Fig. 1A and B, only online). The PIK3CD mutation (E1021K, heterozygous) was detected using diagnostic exome sequencing and confirmed via Sanger sequencing. Sirolimus treatment was initiated after APDS1 diagnosis at $2 \mathrm{mg}$ per day $\left(2.9 \mathrm{mg} / \mathrm{m}^{2}\right)$, increasing to 2.5 $\mathrm{mg}\left(3.6 \mathrm{mg} / \mathrm{m}^{2}\right)$ after 2 weeks. Corresponding sirolimus trough levels were $4.4-6.9 \mathrm{ng} / \mathrm{mL}$. The cervical lymph nodes almost disappeared after 1 month; however, high-dose IGRT was still required. We increased the sirolimus dosage to $3 \mathrm{mg}$ per day $\left(4.3 \mathrm{mg} / \mathrm{m}^{2}\right)$; however, after 20 days, sirolimus trough levels 
Table 1. Clinical and Immunological Manifestations of APDS1 in Three Patients

\begin{tabular}{|c|c|c|c|}
\hline \multirow{2}{*}{ Age at genetic diagnosis/sex } & Patient 1 & Patient 2 & Patient 3 \\
\hline & 4 yr, male & $4 \mathrm{yr}$, male & 13 yr, female \\
\hline Family history of PID & $\mathrm{N}$ & $\mathrm{N}$ & $\mathrm{N}$ \\
\hline \multicolumn{4}{|l|}{ Infectious complications } \\
\hline BCGiosis & $\mathrm{N}$ & $\sqrt{ }(3$ month) & $\sqrt{ }$ (unknown) \\
\hline Recurrent respiratory infections & $\sqrt{ }$ (infant) & $\sqrt{ }$ (infant) & $\sqrt{ }$ (infant) \\
\hline CMV colitis & $\sqrt{ }(4 \mathrm{yr})$ & $\sqrt{ }(4 \mathrm{yr})$ & $\mathrm{N}$ \\
\hline Tonsillitis (with tonsillectomy) & $\sqrt{ }$ (43 month) & $\mathrm{N}$ & $\mathrm{N}$ \\
\hline Osteomyelitis & $\mathrm{N}$ & $\mathrm{N}$ & $\sqrt{ }(4 \mathrm{yr})$ \\
\hline \multicolumn{4}{|l|}{ Non-infectious complications } \\
\hline Lymphadenopathy & $\sqrt{ }$ (27 month) & $\sqrt{ }(38$ month) & $\sqrt{ }(4 \mathrm{yr})$ \\
\hline Splenomegaly & $\sqrt{ }(4 \mathrm{yr})$ & $\sqrt{ }$ (43 month) & $\sqrt{ }(10 \mathrm{yr})$ \\
\hline Hepatomegaly & $\mathrm{N}$ & $\mathrm{N}$ & $\sqrt{ }(10 \mathrm{yr})$ \\
\hline Cytopenia & $\sqrt{ }(10$ month $)$ & $\sqrt{ }(2$ month $)$ & $\mathrm{N}$ \\
\hline Nodular mucosal lymphoid hyperplasia of the intestine & $\sqrt{ }(4 \mathrm{yr})$ & $\sqrt{ }$ (43 month) & $\sqrt{ }(6 \mathrm{yr})$ \\
\hline Hematochezia/diarrhea & $\sqrt{ }(17$ month) & $\sqrt{ }(50$ month) & $\sqrt{ }(6 \mathrm{yr})$ \\
\hline Poor weight gain & $5-10 p$ & $25-50 p$ & $3 p$ \\
\hline Macrocrania (> 95 p) & $\sqrt{ }$ & $\sqrt{ }$ & $\mathrm{N}$ \\
\hline Lymphoma & $\mathrm{N}$ & $\mathrm{N}$ & $\mathrm{N}$ \\
\hline Bronchiectasis & $\mathrm{N}$ & $\mathrm{N}$ & $\sqrt{ }$ \\
\hline Pulmonary nodular infiltration & $\mathrm{N}$ & $\sqrt{ }$ (43 month) & $\sqrt{ }(10 \mathrm{yr})$ \\
\hline \multicolumn{4}{|l|}{ Immunological workup } \\
\hline T\&B lymphocyte subsets & $\begin{array}{c}(4 \mathrm{yr}) \\
\text { CD3 } \mathrm{nl} \\
\mathrm{CD} 4 \downarrow \\
\text { CD8 } \uparrow \\
\text { CD4/CD8 inverted } \\
\text { CD19 } \mathrm{nl}\end{array}$ & $\begin{array}{c}(4 \mathrm{yr}) \\
\mathrm{CD} 3 \mathrm{nl} \\
\mathrm{CD} 4 \downarrow \\
\mathrm{CD} 8 \uparrow \\
\text { CD4/CD8 inverted } \\
\text { CD19 } \mathrm{nl}\end{array}$ & $\begin{array}{c}(12 \text { yr) } \\
\text { CD3 } \downarrow \\
\text { CD4 } \downarrow \\
\text { CD8 } \mathrm{nl} \\
\text { CD4/CD8 inverted } \\
\text { CD19 } \downarrow\end{array}$ \\
\hline PHA stimulation* & Decreased (4 yr) & sl. Decreased (4 yr) & $\begin{array}{c}\mathrm{nl}(4 \mathrm{yr}) \\
\text { absent (12 yr) }\end{array}$ \\
\hline CD16 CD56 cell count & $\mathrm{nl}(4 \mathrm{yr})$ & $\mathrm{nl}(4 \mathrm{yr})$ & $\mathrm{nl}(12 \mathrm{yr})$ \\
\hline Immunoglobulins & $\lg G \downarrow \downarrow, \lg A \downarrow, \lg M \uparrow(4 \mathrm{yr})$ & $\lg G \downarrow \downarrow, \lg A \downarrow, \lg M \uparrow(4 \mathrm{yr})$ & $\lg G \uparrow \uparrow, \lg A \downarrow, \lg M \uparrow(12 \mathrm{yr})$ \\
\hline
\end{tabular}

The numbers in parentheses indicate the age of symptom onset or the age at the laboratory test.

$\checkmark$, present; $\uparrow$, increased abundance; $\downarrow$, decreased abundance; nl, normal; N, none; APDS, activated phosphoinositide 3-kinase $\delta$ syndrome; PID, primary immunodeficiency disease; BCGiosis, Bacillus Calmette-Guérin vaccine site infection; CMV, cytomegalovirus; PHA, phytohemagglutinin; $p$, percentile.

*Because of laboratory limitations, QuantiFERON-TB Gold Tests were used.

abruptly rose to $14.7 \mathrm{ng} / \mathrm{mL}$, and serum creatinine levels rose from baseline 0.28 to $0.45 \mathrm{mg} / \mathrm{dL}$. After 3 days of withdrawal, the trough level decreased to $9.6 \mathrm{ng} / \mathrm{mL}$, with normalization of creatinine levels. Sirolimus was restarted at a dose of $2.5 \mathrm{mg}$ and maintained. Currently, the patient's IgG trough levels remain above $400 \mathrm{mg} / \mathrm{dL}$ with IGRT (500-600 mg/kg every 4 weeks). Significant improvement of multiple lymphoid hyperplasia was noted after 6 months (Fig. 1D-H). However, a colon biopsy revealed CMV, and the patient complained of diarrhea (Supplementary Fig. 1C, only online). Consequently, VGCV was administered for 6 weeks. Presently, there is no evidence of bone marrow suppression or mucositis and no infectious complications requiring hospitalization.

A 13-year-old female (Patient 3) previously diagnosed with selective IgA deficiency was reevaluated for other PIDs when she presented with multiple episodes of prolonged fever and enlargement of several lymph nodes. Patient 3 had a history of hospitalizations attributable to recurrent respiratory tract infections, cellulitis, and osteomyelitis. She was diagnosed with selective IgA deficiency at the age of 4 years at our hospital. Cervical lymph node enlargement was first noticed at the age of 6 years; hematochezia was also noted. Colon and cervical lymph node biopsies showed atypical lymphocyte proliferation with polyclonality, and persistent atypical lymphoid proliferation of the colon was observed at the age of 10 years. One colon biopsy specimen was suspicious for mucosa-associated lymphoid tissue lymphoma. The oncologists decided to observe the patient without special chemotherapy. During the follow-up period, we diagnosed and treated Patient 1 from the present case series, leading the clinicians to reevaluate Patient 3 for other PIDs. Upon reevaluation, the height and weight of Patient 3 were below the 5 th percentile and development was 

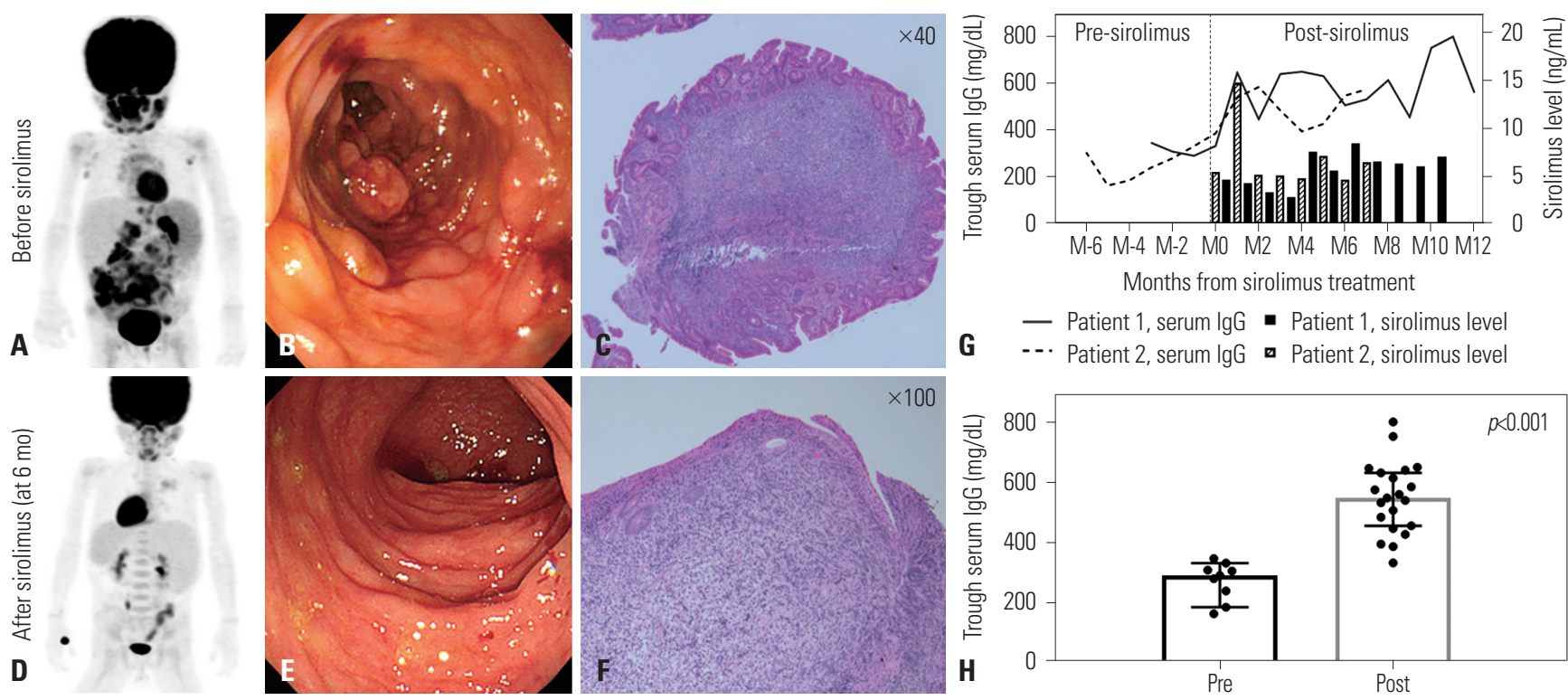

Fig. 1. Comparison of the clinical features and histologic findings before and after treatment with sirolimus (Patient 2) and trough immunoglobulin (Ig)G levels before and after sirolimus treatment (Patient 1 and Patient 2). (A-F) Positron emission tomography/computed tomography (PET/CT), colonoscopy, and colon biopsy findings before and after 6 months of treatment with sirolimus in Patient 1. PET/CT revealed enlargement of multiple lymph nodes, including bilateral cervical lymph nodes (A), and colonoscopy revealed multiple nodules and masses throughout the entire length of the colon (B). Biopsy of the colon specimen showed lymphoid cell aggregates and surface erosion (C). After 6 months of treatment with sirolimus, the uptake observed in the lymph nodes and spleen on PET/CT almost disappeared (D), and lymphoid aggregations in the colon were significantly improved (E) and (F). Changes in IgG and sirolimus trough levels following treatment with sirolimus are shown (G). The lgG trough level after sirolimus treatment (median, $594 \mathrm{mg} / \mathrm{dL}$; range, $332-799 \mathrm{mg} / \mathrm{dL}$ ) was significantly higher than before sirolimus treatment (median, $290 \mathrm{mg} / \mathrm{dL}$; range, 163-346 mg/dL) $(p<0.001)(\mathrm{H})$.

normal. At the age of 12 years, laboratory values were $\mathrm{WBC}$, $5410 / \mu \mathrm{L} ; \mathrm{Hb}, 8.5 \mathrm{~g} / \mathrm{dL}$; and PLT, 285k/ $\mu \mathrm{L}$. Immunological values were CD3, 391/ $\mu \mathrm{L}$ (normal, 800-3500/ $\mu \mathrm{L}$ ); CD4, 194/ $\mu \mathrm{L}$ (normal, 400-2100/ $\mu \mathrm{L}$ ); CD8, 190/ $\mu \mathrm{L}$ (normal, 200-1200/ $\mu \mathrm{L}$ ); and CD19, $54 / \mu \mathrm{L}$ (normal, 200-600/ $\mu \mathrm{L}$ ). The CD4:CD8 ratio was 1.02. The response to phytohemagglutinin mitogen was reduced, while results from the same test performed 8 years prior were normal. Serum IgG, A, and M levels were $1920 \mathrm{mg} /$ $\mathrm{dL}$ (normal, 639-1349 mg/dL), $3 \mathrm{mg} / \mathrm{dL}$ (normal, 70-312 mg/ $\mathrm{dL}$ ), and $1138 \mathrm{mg} / \mathrm{dL}$ (normal, 56-352 mg/dL), respectively. Hypermetabolic enlarged lymph nodes, splenomegaly, and bronchiectatic changes in the middle lobe of the right lung were observed on PET/CT. Diagnostic exome sequencing revealed a heterozygous E1021K mutation in PIK3CD, which was confirmed via Sanger sequencing. Sirolimus was initiated at 3 $\mathrm{mg}$ per day $\left(2.55 \mathrm{mg} / \mathrm{m}^{2}\right)$, with trough levels of $4.6-6.7 \mathrm{ng} / \mathrm{mL}$. There were no adverse events, the hematochezia improved, and the size of the lymph nodes decreased. Patient 3 was in the 50-75th percentile for body weight and height at the age of 5 years, but had poor growth from 5 to 13 years of age. However, she rapidly gained weight with sirolimus treatment $(2 \mathrm{~kg}$ between 12-13 years before treatment compared to $6 \mathrm{~kg}$ between 13-14 years after treatment), and height percentile changes were observed ( $<3$ rd percentile before to $5-10$ th percentile after treatment) (Supplementary Fig. 2, only online).

This is the first case series of sirolimus treatment in Korean patients with APDS1. We have described the detailed clinical responses to sirolimus treatment, which will help other clini- cians who try this therapeutic option. Some adverse events (elevation of serum creatinine at higher doses, neutropenia, and stomatitis) were observed, although they were manageable.

Patients with APDS can exhibit numerous clinical manifestations at various ages. ${ }^{1,2}$ In 53 APDS patients from a large cohort study, ${ }^{2}$ recurrent respiratory tract infection was the most frequently observed complication (95\%), followed by lymphadenopathy (64\%), bronchiectasis (60\%), and splenomegaly (58\%). Enteropathy (25\%) and nodular mucosal lymphoid hyperplasia $(32 \%)$ were observed in a relatively small portion of patients. All 3 patients in our series had intestinal nodular mucosal lymphoid hyperplasia. Patient 2 showed abnormal uptake on PET/ CT in the intestine, and massive nodular mucosal lymphoid hyperplasia was detected, although he had no gastrointestinal symptoms. Therefore, physicians may consider an initial gastrointestinal endoscopic evaluation in APDS1 patients regardless of symptoms.

Activation of PI3K/Akt/mTOR signaling leads to lymphoid proliferation via the nuclear factor kappa B pathway; therefore, inhibiting PI3K overexpression or its downstream pathway may help alleviate the disease course ${ }^{5-7}$ Sirolimus, everolimus, and temsirolimus, as well as leniolisib have been introduced as pathway-based targeted therapies in patients with APDS. ${ }^{2,89}$ Recently, a study of 77 APDS patients reported that lymphoproliferation responded well to sirolimus in 26 patients, with less response to gut inflammation and cytopenia: there was no mention of hypogammaglobulinemia or changes in IGRT requirement (dose or intervals). ${ }^{10}$ All three patients in our study received si- 
rolimus, and their symptoms responded well to treatment. The immunoglobulin dose was reduced in two patients requiring high-dose, short interval regular IGRT. Trough IgG levels were significantly higher after sirolimus treatment than before (Fig. $1 \mathrm{G}$ and $\mathrm{H}$ ). Improvements in enteropathy leading to proteinlosing gastroenteropathy possibly explains the reduced severity of hypogammaglobulinemia. Diarrheal symptoms significantly improved upon sirolimus treatment, while low IgA and high IgM levels were unchanged. Although IGRT doses and intervals were improved (Patients 1 and 2), IGRT could not be discontinued.

Safety and efficacy data for sirolimus in APDS patients are limited. The median trough level of sirolimus in our patients was on the lower side of the range used to prevent graft rejection in kidney or heart transplant recipients (4-12 ng/mL). ${ }^{11}$ Two patients in this series experienced adverse events. Patient 1 suffered from granulocytopenia and oral stomatitis, and Patient 2 experienced elevations in serum creatinine with an abrupt surge in sirolimus trough levels when increasing the dose. Complications from treatment with sirolimus have also been reported elsewhere, ${ }^{10}$ in which four patients completely stopped the medication due to poor compliance (2 patients) and headache, vomiting, and nephrotoxicity (2 patients). Further, three patients temporarily stopped sirolimus attributable to aphthous ulcer, hepatotoxicity, and renal toxicity. A lower target range of sirolimus has been suggested for APDS patients than for solid organ transplant recipients. ${ }^{8}$ We propose the use of sirolimus with a lower range of trough levels alongside precise monitoring of blood cell counts and renal function to avoid renal toxicity and other complications, including possible opportunistic infections related to neutropenia. The efficacy and safety of longterm sirolimus use in patients with APDS remain uncertain, and the role of sirolimus in APDS patients with complications of malignant tumors, such as lymphoma, is also unclear. Sirolimus treatment in APDS patients should be followed long term.

In conclusion, we report three patients with APDS1 for the first time in Korea. A low-dose mTOR inhibitor might relieve severe hypogammaglobulinemia, as well as lymphoproliferation and enteropathy, without major adverse effects in patients with APDS1.

\section{ACKNOWLEDGEMENTS}

We thank the patients and their families for their participation in this study. This work was partially supported by a research grant from The Korean Society of Pediatric Infectious Diseases, 2016. This research was also supported by the Basic Science Research Program through the National Research Foundation of Korea funded by the Ministry of Education (2019032869).

\section{AUTHOR CONTRIBUTIONS}

Conceptualization: Ji-Man Kang, Su Kyung Kim, and Yea-Jean Kim.
Data curation: Dongsub Kim, Sae Rom Choi, Yeon Jung Lim, Soon Ki Kim, Byung-Kiu Park, Yon Ho Choe, and Ji Won Lee. Formal analysis: Ji-Man Kang, Su Kyung Kim, and Yea-Jean Kim. Investigation: Ji-Man Kang, Su Kyung Kim, and Yea-Jean Kim. Methodology: Ji-Man Kang, Su Kyung Kim, and Yea-Jean Kim. Supervision: Yae-Jean Kim. Validation: Yae-Jean Kim. Visualization: Weon Seo Park, Eun-Suk Kang, and Young Hyeh Ko. Writing_original draft: Ji-Man Kang and Su Kyung Kim. Writing_review \& editing: Ji-Man Kang, Su Kyung Kim, and Yae-Jean Kim. Approval of final manuscript: all authors.

\section{ORCID iDs}

Ji-Man Kang Su Kyung Kim Dongsub Kim

Sae Rom Choi Yeon Jung Lim Soon Ki Kim

Byung-Kiu Park Weon Seo Park Eun-Suk Kang Young Hyeh Ko Yon Ho Choe Ji Won Lee Yae-Jean Kim https://orcid.org/0000-0002-0678-4964 https://orcid.org/0000-0002-3204-1398 https://orcid.org/0000-0002-9836-6769 https://orcid.org/0000-0002-5839-6218 https://orcid.org/0000-0002-8815-333X https://orcid.org/0000-0002-4785-1705 https://orcid.org/0000-0001-9743-0610 https://orcid.org/0000-0002-0035-1455 https://orcid.org/0000-0001-6386-6520 https://orcid.org/0000-0002-4383-0579 https://orcid.org/0000-0003-1525-7688 https://orcid.org/0000-0003-0084-1304 https://orcid.org/0000-0002-8367-3424

\section{REFERENCES}

1. Lucas CL, Kuehn HS, Zhao F, Niemela JE, Deenick EK, Palendira U, et al. Dominant-activating germline mutations in the gene encoding the PI(3)K catalytic subunit p110 $\delta$ result in T cell senescence and human immunodeficiency. Nat Immunol 2014;15:88-97.

2. Coulter TI, Chandra A, Bacon CM, Babar J, Curtis J, Screaton N, et al. Clinical spectrum and features of activated phosphoinositide 3-kinase $\delta$ syndrome: a large patient cohort study. J Allergy Clin Immunol 2017; 139:597-606.e4.

3. Bravo García-Morato M, García-Miñaúr S, Molina Garicano J, Santos Simarro F, Del Pino Molina L, López-Granados E, et al. Mutations in PIK3R1 can lead to APDS2, SHORT syndrome or a combination of the two. Clin Immunol 2017;179:77-80.

4. Deau MC, Heurtier L, Frange P, Suarez F, Bole-Feysot C, Nitschke $\mathrm{P}$, et al. A human immunodeficiency caused by mutations in the PIK3R1 gene. J Clin Invest 2014;124:3923-8.

5. Pongas G, Cheson BD. PI3K signaling pathway in normal B cells and indolent B-cell malignancies. Semin Oncol 2016;43:647-54.

6. Vivanco I, Sawyers CL. The phosphatidylinositol 3-Kinase AKT pathway in human cancer. Nat Rev Cancer 2002;2:489-501.

7. Pauls SD, Lafarge ST, Landego I, Zhang T, Marshall AJ. The phosphoinositide 3-kinase signaling pathway in normal and malignant B cells: activation mechanisms, regulation and impact on cellular functions. Front Immunol 2012;3:224.

8. Rae W, Ramakrishnan KA, Gao Y, Ashton-Key M, Pengelly RJ, Patel SV, et al. Precision treatment with sirolimus in a case of activated phosphoinositide 3-kinase $\delta$ syndrome. Clin Immunol 2016;171:38-40.

9. Rao VK, Webster S, Dalm VASH, Šedivá A, van Hagen PM, Holland $S$, et al. Effective "activated PI3K $\delta$ syndrome"-targeted therapy with the PI3K $\delta$ inhibitor leniolisib. Blood 2017;130:2307-16.

10. Maccari ME, Abolhassani H, Aghamohammadi A, Aiuti A, Aleinikova O, Bangs C, et al. Disease evolution and response to rapamycin in activated phosphoinositide 3-kinase $\delta$ syndrome: The European Society for Immunodeficiencies-Activated Phosphoinositide 3-Kinase $\delta$ Syndrome Registry. Front Immunol 2018;9:543.

11. De Simone P, Fagiuoli S, Cescon M, De Carlis L, Tisone G, Volpes $\mathrm{R}$, et al. Use of everolimus in liver transplantation: recommendations from a working group. Transplantation 2017;101:239-51. 